\title{
Dynamic Vulnerability Analysis of Mountain Settlements Exposed to Geological Hazards: A Case Study of the Upper Min River, China
}

\author{
Mingtao Ding $\mathbb{D}^{1,2}$ Chuan Tang $\mathbb{D}^{2},{ }^{2}$ Tao Huang $\mathbb{D},{ }^{1}$ and Zemin Gao $\mathbb{D}^{1}$ \\ ${ }^{1}$ Faculty of Geosciences and Environmental Engineering, Southwest Jiaotong University, Chengdu 611756, China \\ ${ }^{2}$ State Key Laboratory of Geohazard Prevention and Geoenvironment Protection, Chengdu University of Technology, \\ Chengdu 610059, China
}

Correspondence should be addressed to Mingtao Ding; mingtaoding@163.com and Chuan Tang; 2401634274@qq.com

Received 30 May 2020; Revised 15 July 2020; Accepted 7 August 2020; Published 28 August 2020

Academic Editor: Chong Xu

Copyright (C) 2020 Mingtao Ding et al. This is an open access article distributed under the Creative Commons Attribution License, which permits unrestricted use, distribution, and reproduction in any medium, provided the original work is properly cited.

The upper reaches of Min River (The upper Min River) is located in Southwest China with significant mountain settlements, which are vulnerable to frequent geological hazards. Based on a field investigation, collation of yearbook data, and analysis through the use of SPSS statistical software, a vulnerability evaluation index system of geological hazards was devised. According to the actual field situation and the acquired data of the study area in 2006, 2009, and 2015, 16 indicators were selected as settlement vulnerability evaluation indexes of geological hazards. The indexes included population density, building coverage, and economic density. Based on the comprehensive evaluation model of entropy value, the dynamic change in the settlement vulnerability of geological hazards was analyzed. The results showed that population density, building coverage, economic density, and road density were the factors that affected the settlement vulnerability of geological hazards the most-Wenchuan earthquake caused considerable damage to the upper Min River, making the area the most vulnerable in 2009. However, its vulnerability decreased in 2015, which indicated that postearthquake reconstruction achieved significant results. Thus, the vulnerability has emerged as an important indicator reflecting the safety and healthy development of mountain settlements.

\section{Introduction}

The vulnerability assessment of geological hazards is a necessary process of regional risk analysis. Furthermore, the vulnerability of geological hazards fluctuates with time. The upper Min River, as a topographic uplift and economic valley area, is in the process of rapid construction of social structure after the settlement was devastated by the massive Wenchuan earthquake of 2008 [1]. Therefore, the dynamic assessment of mountain settlement vulnerability of geological hazards in the upper Min River is an essential part of the mechanism for regional disaster risk reduction, prevention, and capacity building for emergencies.

In 1848, John Stuart Mill pointed out in his book, the Principles of political economy with some of their applications to social philosophy, that all signs of destruction caused by earthquakes, floods, hurricanes, and wars would disappear in a short time and that the country would recover rapidly from the state of disasters, indicating that human and social factors were important aspects affecting disaster risk $[2,3]$. As a result, it was gradually realized that improving social disaster-bearing and emergency response capacity was more accessible than changing disaster-causing factors which further helped people understand the importance of vulnerability to disaster research. For natural disasters, the important thing is not to explain the characteristics, consequences, and causes of disasters by the conditions or the actions of disasters but to analyze the contemporary social order, assess the daily relations of disasters, and shape the historical environment of these characteristics [4]. In addition, socioeconomic factors, such as population characteristics, industrial structure, and spatial distribution, have 
an impact on natural disasters. Therefore, vulnerability is not only a central concept in the field of disaster research but also a means to solve the problems related to population, development, and environment $[5,6]$.

At present, vulnerability research has developed rapidly - not only as qualitative research but also as quantitative research. Guo [7] analyzed the relationship between natural disasters and social vulnerability, believing that vulnerability includes material and spiritual aspects and that there is a close relationship between social vulnerability and disaster types, disaster nature, disaster intensity, and disaster occurrence time. Hu et al. [8] analyzed the impact of social factors on disasters, pointing out that social factors mainly affect disasters by influencing the source of disasters, the ability of disaster-bearing bodies to prevent and resist disasters, and the level of social and economic development. Liu et al. $[9,10]$ divided vulnerability evaluation factors into property index and population index. He put forward the conversion assignment function, which solved the problem of unified scaling and comprehensive expression of human, financial, and material aspects and discussed the vulnerability calculation method. Pang et al. [11] analyzed five parts of dynamic risk assessment of natural disasters: time constraints, disaster-causing factors, disaster-bearing bodies, coupling, and scenario output. They also gave a basic model of dynamic risk assessment of natural disasters in a narrow sense.

Meanwhile, many scholars have made vulnerability analyses based on a large number of cases. Taking Kangding County as an example, Wang et al. [12] put forward a new method for evaluating the socioeconomic vulnerability of urban geological hazards and established a vulnerability evaluation system. Tang [13] selected population density, housing property, GDP, and cultivated land and highway distribution as vulnerability evaluation factors of landslide disasters in compiling the landslide risk map of the Red River Basin. Wu et al. [14] selected housing construction, road engineering, lifeline engineering, population distribution, and land use as vulnerability assessment factors in landslide hazard risk assessment in the new urban area of Badong County. Miao and Ding [15] selected eight factors as evaluation indexes. The factors were population density, building coverage, road density, number of hospitals per 10,000 persons, number of welfare institutes per 10,000 persons, number of village committees per 10,000 persons, mobile users' proportion at the end of the year, and number of schools per 10,000 persons. They used the entropy method to obtain the vulnerability zoning map of the Lushan earthquake area.

Vulnerability research has become an indispensable part of natural disaster risk research [16]. However, the overall research still relates mainly to the analysis of the natural factors causing disasters, and there is still a lack of correct understanding and in-depth discussion of the social factors involved in disasters.

This study chooses the mountain settlements in the upper Min River as the research object. It uses the entropy method to carry out the dynamic assessment of the vulnerability of mountain settlements to geological hazards under time constraints and obtains the zoning maps of the vulnerability in different periods. Thus, the study provides an essential scientific basis for the compilation of data on regional geological hazards, planning risk prevention, and the formulation of emergency measures.

\section{Study Area}

The upper Min River is situated between $30^{\circ} 45^{\prime} \mathrm{N}$ to $33^{\circ} 10^{\prime} \mathrm{N}$ and $102^{\circ} 35^{\prime} \mathrm{E}$ to $103^{\circ} 57^{\prime} \mathrm{E}$. This area belongs to the uplift area of China's topography and the active belt of the tectonic movement. The occurrence of several strong earthquakes in the region has resulted in the loosening of the surface material, damage to deep rocks and the soil mass, and the decrease in water saturation rate in the study area $[17,18]$. All these factors have led to frequent secondary disasters in the area, such as topples, slides, and flows. The paper mainly studies the dynamic vulnerability analysis of mountain settlements and does not involve unmanned areas. Therefore, it only selects the geological disaster points that bring harm to human survival or damage the human living environment. The geoenvironmental information system of Sichuan Province [19] stores such data and does not involve geological phenomena such as slides and flows in unmanned areas. According to the geoenvironmental information system of Sichuan province, there have been 2,778 geological hazards in the study area. According to the new classification system proposed by Hunger et al. [20], these geological hazards include 803 slides, 726 topples, 770 flows, and 479 slopes deformation (Figure 1). The study area is inhabited by 22 ethnic groups, such as Tibet, Qiang, Hui, and Han.

Moreover, the area is home to the largest Qiang ethnic community in China. Its ethnic culture has distinctive characteristics of mixed cohabitation. Besides, under the influence of the farming culture of the Central Plains and the traditional Qiang-Tibetan culture, the region's economy is still relatively backward [21].

\subsection{Distribution and Evolution of Mountain Settlements.} The spatial pattern of settlements in the upper Min River has changed significantly from 2006 to 2015 . Its distribution is manifested mainly by the migration of the settlements with time from high altitude areas and large slope areas to the river valley platforms. The trend indicated a shift from decentralization to agglomeration. Consequently, the settlement area gradually decreased from $955 \mathrm{~km}^{2}$ in 2006 to $462 \mathrm{~km}^{2}$ in 2015 (Figure 2).

\section{Materials and Methods}

3.1. Division of Cell Meshes. The primary data of this evaluation are based on the township data, and the operation and processing of the data are determined from the division of regional grid cells. This is done due to the apparent advantage of using grid cells in spatial data superposition calculation. The division of grid size directly affects the rationality of evaluation results. At present, the selection of grid size depends mainly on the resolution of original data and the experience and knowledge of the experts. 


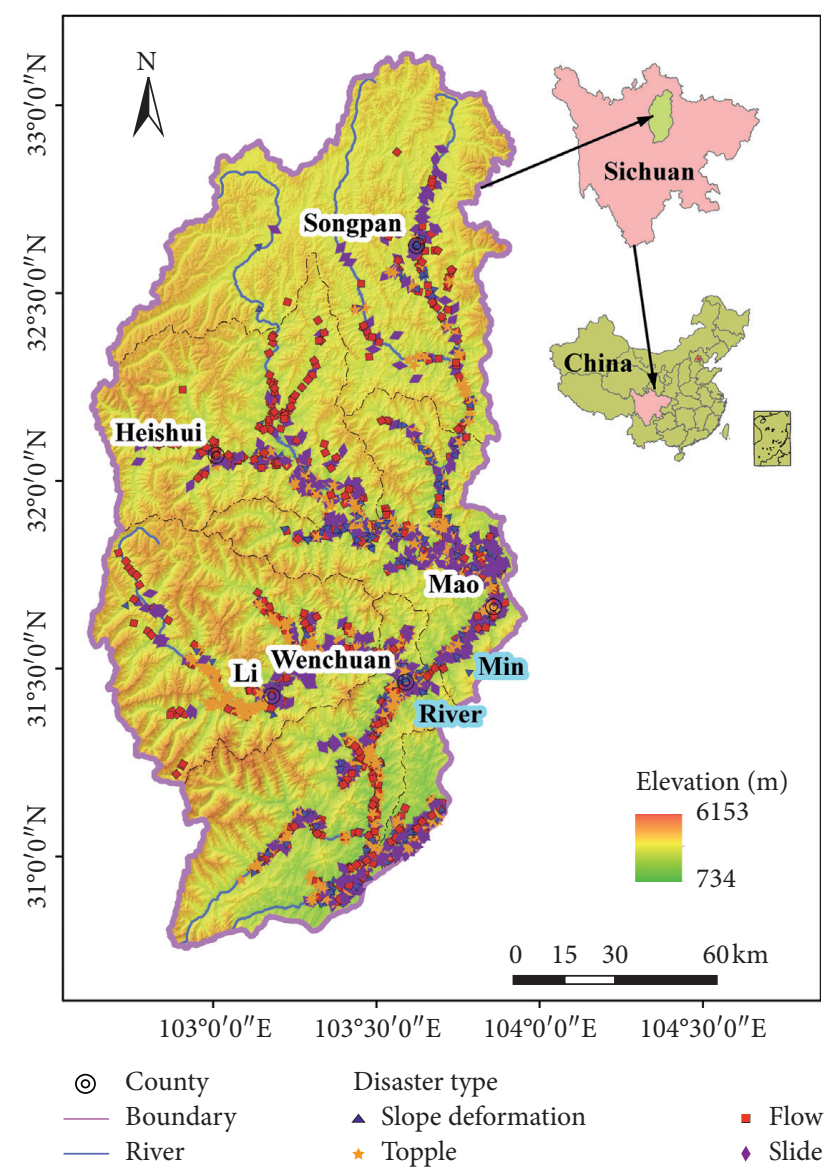

Figure 1: Location of the upper Min River (inset map shows Sichuan and China) and the distribution of geological disasters in the study area.

"Technical Guide for Evaluation of Resources and Environment Carrying Capacity and Suitability of Land and Space Development" (for trial implementation) [22] proposes to use a grid with a spatial resolution of $50 \times 50 \mathrm{~m}$ at the regional scale. Therefore, the vulnerability assessment unit of this study adopts a $50 \times 50 \mathrm{~m}$ resolution grid unit.

3.2. Construction of the Evaluation Index System. The index system of vulnerability assessment includes population, economy, society, and other factors, which referred to the existing research results on disaster vulnerability and other related index systems in mountain areas [23-26]; 43 evaluation indicators were selected from the aspects of population characteristics, social security, social economy, structural characteristics, and natural characteristics. The evaluation index system for the settlement vulnerability of geological hazards in the upper Min River was devised at four levels: target level, criterion level, element layer, and index level (Table 1).

The vulnerability evaluation indexes of geological hazards are different at different scales. The social and economic development of the upper Min River is reduced, and data collection is complicated. In the selection of indicators, we must not only follow the scientific principle but also fully consider the reality to ensure the availability of data. 27 indexes were excluded because they could not collect the complete time series data. 16 indexes are selected as vulnerability evaluation factors of the geological disasters in the upper Min River. These indexes include property threatened by geological disasters (D1), number of people threatened by geological disasters (D2), population density (D3), hazard density (D4), teacher-student ratio (D5), economic density (D6), GDP ratio of primary industry (D7), building coverage (D8), road density (D9), number of village committees per 10,000 persons (D10), per capita GDP (D11), per capital investment in fixed assets (D12), general public budget expenditure (D13), per capita mobile phones (D14), number of medical technicians per 10,000 persons (D15), and engineering protection (D16).

The basic data of this study are obtained mainly from the following three aspects:

(1) Database on the potential points of geological hazards: According to the geoenvironmental information system of Sichuan province [19] (http://202.61. 89.33:16003), information related to coordinates, geographic location, direction, volume, scale, property threatened by geological disasters, number of people threatened by geological disasters, number of households threatened by geological disasters, and prevention engineering of the potential geological hazards in the upper Min River since 2008 was obtained.

(2) Remote sensing images and vector data: Based on the National Earth System, science data-sharing infrastructure [27] (http://www.geodata.cn), the enhanced thematic mapper (ETM) image of the study area, and the essential data related to fields like topography, land use, and earthquakes were obtained.

(3) Data from yearbooks and statistical bulletins: The data on administrative areas, economic output, a permanent population, practitioners of secondary and tertiary industries, industrial output, built-up areas of towns and townships, and other related data were obtained from several yearbooks and statistical bulletins for the years 2006 to 2016. The sources included "China County Economic Statistical Yearbook," "Sichuan Statistical Yearbook," "Aba Statistical Yearbook," "Chengdu Statistical Yearbook," "Heishui Statistical Yearbook," "Wenchuan Statistical Yearbook," "Mao Statistical Yearbook," "Songpan Statistical Yearbook," and "Li Statistical Yearbook." The data not included in yearbooks and statistical bulletins were obtained through field investigation. For the readjustment of the administrative division of Dujiangyan City, at the end of 2014, Zipingpu Town and Hongkou Town were merged into Longchi Town. However, since statistical data are still divided into the yearbooks for three townships, this article does not make any adjustment to it. 


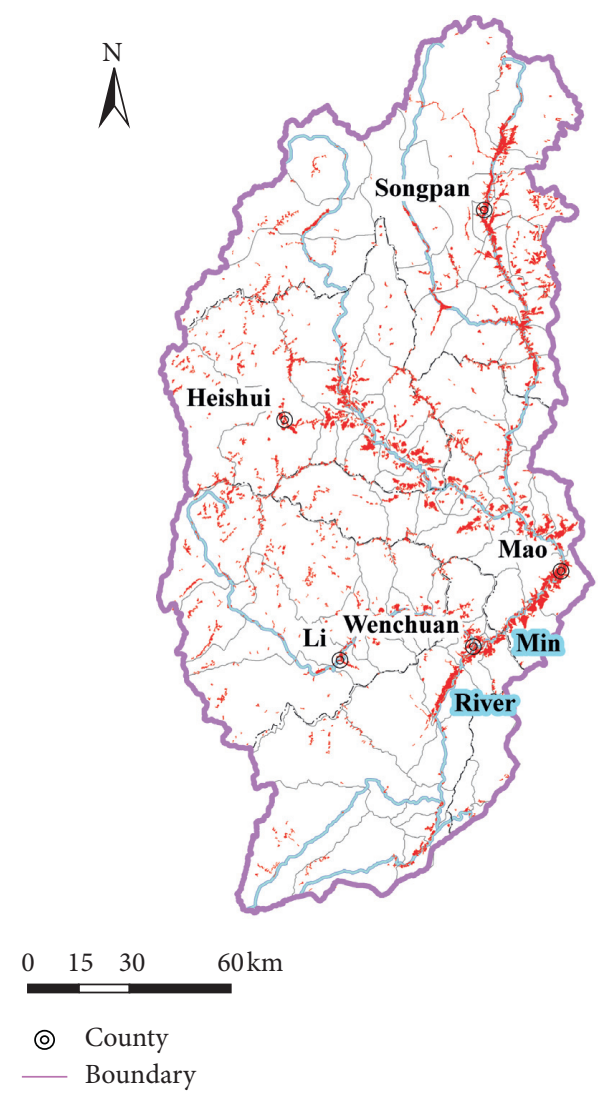

(a)

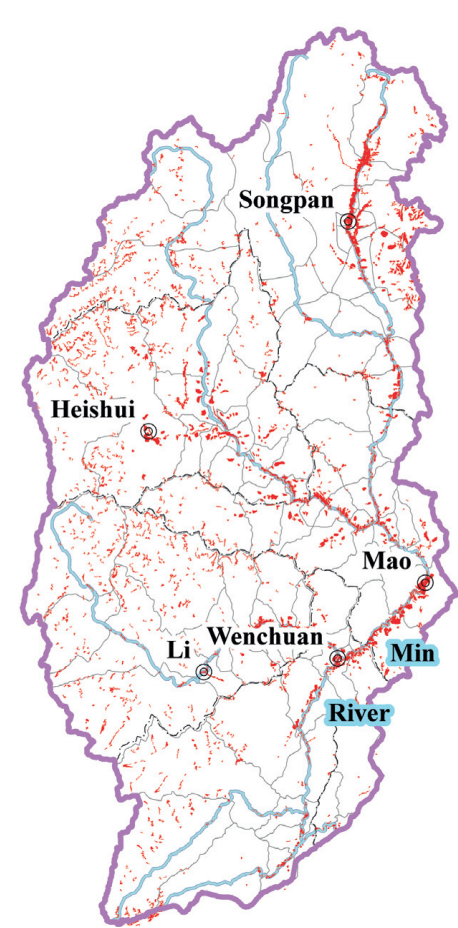

River

(b)

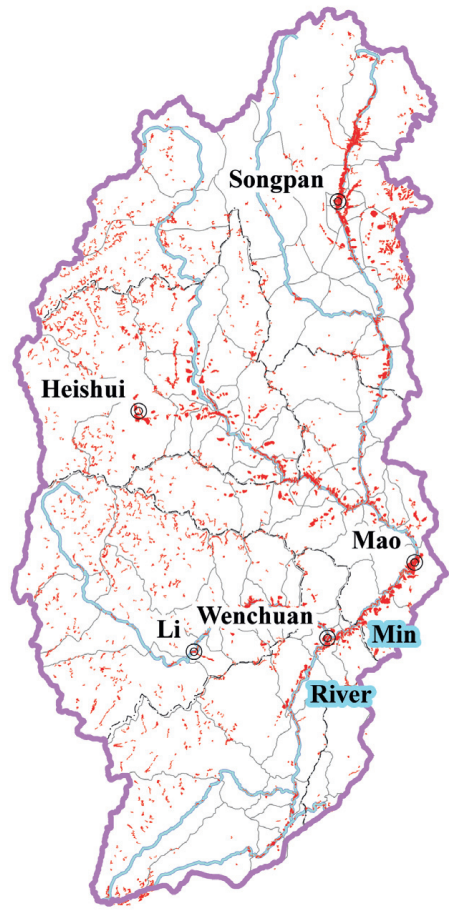

(c)

FIgURe 2: Distribution of mountain settlements in the upper Min River for different years. (a) 2005. (b) 2009. (c) 2015.

The indices selected for vulnerability assessment were defined as follows:

(1) Property threatened by geological disasters (D1): geological disaster threatening property refers to the amount of property that may be lost after a geological disaster occurs. The more threatening property, the more property that may be damaged, and the higher the vulnerability, which is a positive index.

(2) The number of people threatened by geological disasters (D2): the number of people threatened by geological disasters is the number of people threatened by geological disasters in each township. The more people threatened by geological disasters, the more people may be damaged by geological disasters, and the higher the vulnerability, which is a positive index.

(3) Population density (D3): population density is the number of people per unit area. Vulnerability is first related to population density. The higher the population density, the higher the vulnerability of settlements, which is a positive index.

(4) Hazard density (D4): the more geological disaster points, the greater the potential threat to residents and the greater the vulnerability, which is a positive index.
(5) Teacher-student ratio (D5): it is the ratio of students to teachers. The more students assigned by each teacher, the weaker the ability to respond to the crisis. The role of the teacher is very important when disaster strikes. The more teachers are relative to the students in the evacuation and transfer project, the more conducive to ensuring the safety of students' lives, the higher the antirisk ability, and the lower the vulnerability, which is a negative index.

(6) Economic density (D6): economic density refers to the ratio of GDP to the regional area, which characterizes the efficiency of economic activities per unit area of settlements and the intensity of land use. The greater the economic density, the higher the probability of loss in the event of a geological disaster, and the higher the vulnerability of the settlement, which is a positive index.

(7) GDP ratio of primary industry (D7): the GDP ratio of primary industry in the upper Min River mainly refers to agriculture, forestry, animal husbandry, and fisheries. The GDP ratio of the primary industry is an industry with more inputs and less income. Such industries were the leading industries in the upper Min River before 2006, reflecting the society. The higher the degree of closure of the structure, the 
TABLE 1: Vulnerability evaluation index system of the upper Min River.

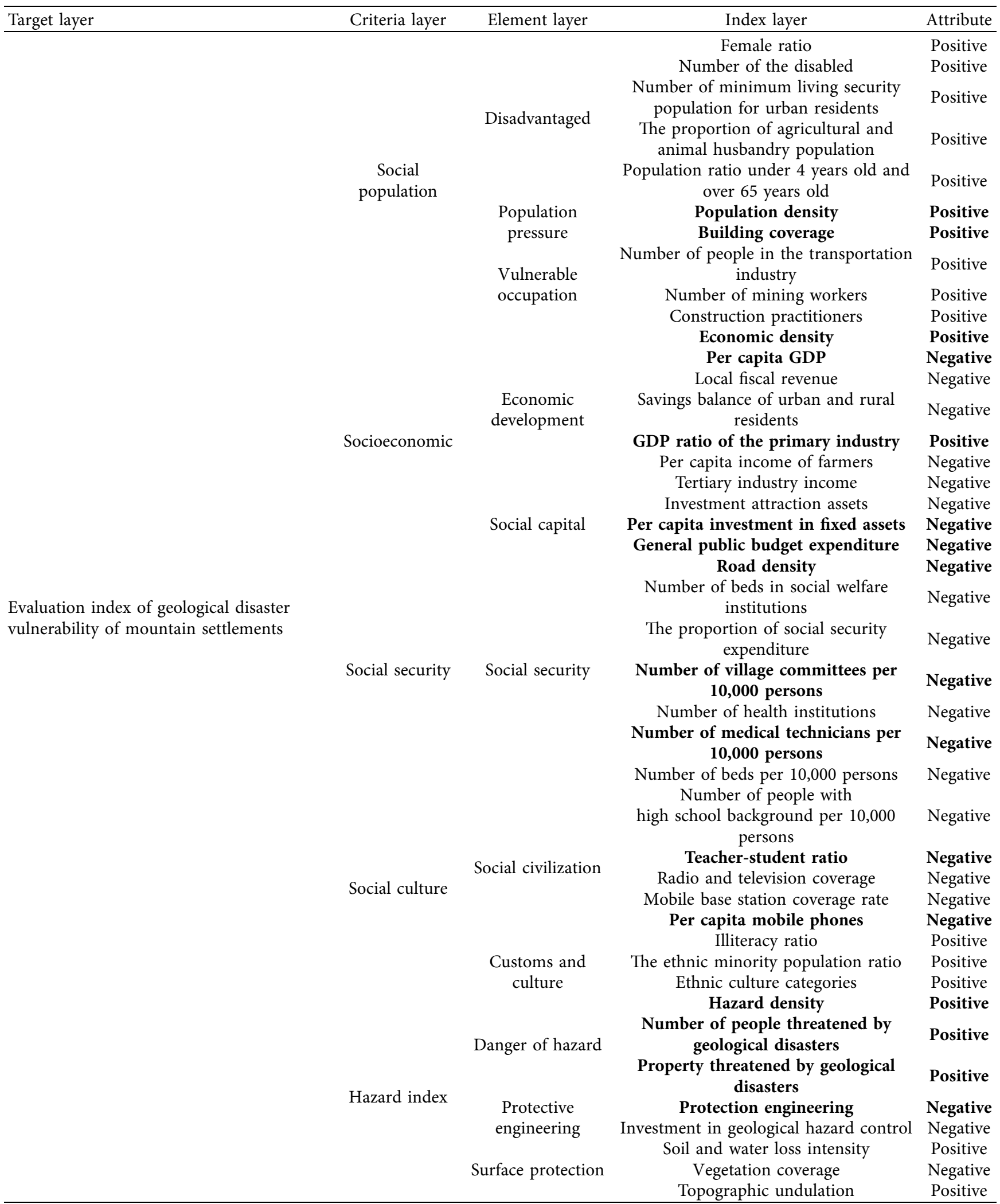

The bold text represents the final selection indexes. 
higher the output value ratio, the higher the vulnerability, which is a positive index.

(8) Building coverage (D8): buildings are also sensitive to geological disasters and are the main disasterbearing bodies of geological disasters in the upper reaches of the Minjiang River. The higher the building coverage, the higher the vulnerability of settlements, which is a positive index.

(9) Road density (D9): road density refers to the ratio of the length of the road in the area to the area of the area, and it reflects the density of roads. The greater the road density, the higher the probability of damage (89-90), and the higher the vulnerability, which is a positive index.

(10) The number of village committees per 10,000 persons (D10): this is the number of village committees owned by every 10,000 people in the study area. Village committees are grassroots mass organizations for self-service, self-management, and self-education of villagers. They are stable and developed in a small area. The primary organization is the grassroots force for the transmission and implementation of national disaster prevention and reduction policies. The more village committees there are, the better the national disaster prevention and mitigation policies are communicated and implemented, and the lower the vulnerability of settlements, which is a negative index.

(11) Per capita GDP (D11): per capita GDP is mainly used to assess the economic development level of a region. At present, there are differences between urban and rural areas in my country. In terms of economic conditions, the financial status of rural residents is worse than that of urban residents. And the more barren a place is, the lower the danger awareness of geological disasters, and the higher the degree of damage when a disaster occurs. And the more personal wealth, the stronger the ability to reconstruct after the disaster, which can reduce the vulnerability of the system to a certain extent. Therefore, the higher the per capita GDP of a region, the lower the vulnerability, which is a negative index.

(12) Per capita investment in fixed assets (D12): per capita investment in fixed assets is the economic activity of construction and purchase of fixed assets, that is, the reproduction of fixed assets. Investment in fixed assets is the primary means of social fixed asset reproduction. Per capita, fixed assets reflect the strength and ability of disaster reconstruction. The greater the per capita investment in fixed assets, the lower the vulnerability, which is a negative index.

(13) General public budget expenditure (D13): it is the expenditure that the region allocates and uses in a planned manner to the centralized budget revenue, and it is the embodiment of the local grassroots construction capacity. The higher the general public budget expenditure, the better the implementation of disaster prevention and mitigation policies, and the lower the vulnerability. It is a negative index.

(14) Per capita mobile phones (D14): the number of mobile phones is an essential index of regional communication capabilities. The more the number of telephones per capita, the faster the transmission of disaster information, which is more conducive to benefit and avoid harm, and the lower the vulnerability. It is a negative index.

(15) The number of medical technicians per 10,000 persons (D15): it indicates that there are medical technicians for every 10,000 people in the study area, and many places in the mountain regions cannot build a medical system. The number of medical technicians can reflect the local rescue ability. The greater the number of doctors, the better the rescue ability after the disaster and the lower the vulnerability of the settlement, which is a negative index.

(16) Engineering protection (D16): protection engineering refers to the number of construction protection projects to control geological disasters. Engineering protection is an effective measure to protect the safety of local residents' lives and property. Therefore, the greater the number of engineering protection, the lower the vulnerability, which is a negative index.

3.3. Evaluation Method. Entropy is an objective, comprehensive evaluation method whose concept originated from thermodynamics [28-31]. It is a measure of uncertainty-the smaller the information entropy of the indicators, the higher the difference coefficient and the weight of the indicators. This means that the information provided by the indicators is large. Thus, it plays an essential role in the comprehensive application. The entropy method is increasingly applied in engineering, management, and social sciences and has gradually become an indispensable practical method in scientific research. Miao and Ding [30] took Lushan and Ludian earthquake-stricken areas as research areas and used the entropy comprehensive evaluation method to determine the social vulnerability of the affected areas. This further proved that the entropy method is practical in social vulnerability assessment. According to the theory and method of information entropy, Ding et al. [32] put forward the concept of "geological hazard entropy" and applied it to the evaluation of geological hazards in the Ranwu-Dongjiu section of Sichuan-Tibet Highway and achieved excellent results.

This study combines the entropy method with geographic information system (GIS) to objectively and intuitively analyze the settlement vulnerability of geological hazards in the upper Min River. The main steps of the entropy comprehensive evaluation method are as follows [31]: 
(a) Normalization of indexes [31]:

Due to the differences in the dimensions, orders of magnitude, units, and quantity changes of the selected indicators in the study, these differences may have an impact on the final analysis results. In order to eliminate these effects, it is necessary to uniformly eliminate the dimensions of the data to solve the problem of the homogenization of qualitative index values. Moreover, because the values of positive and negative indicators represent different meanings (the greater the value of the positive indicator, the greater the vulnerability of mountain settlements, and the smaller the value of the negative indicator, the greater the vulnerability of mountain settlements) Therefore, we use different algorithms to standardize data for positive and negative indicators.

The mountain settlements in the upper Min River are divided into $n$ grids, and $p$ indicators are selected. $x_{i j}$ is the value of the $j$ th indicator of the $i$ th grid unit $(i=1,2, \ldots, n ; j=1,2, \ldots, p)$. The matrix is as follows:

$$
X=\left[\begin{array}{cccc}
x_{11} & x_{12} & \cdots & x_{1 p} \\
x_{21} & x_{22} & \cdots & x_{2 p} \\
\vdots & \vdots & & \vdots \\
x_{n 1} & x_{n 2} & \cdots & x_{n p}
\end{array}\right]=\left(X_{1}, X_{2}, \ldots, X_{p}\right)
$$

When $\mathrm{X}_{i j}$ is a positive indicator [31],

$$
\begin{array}{r}
X_{i j}^{*}=\frac{X_{i j}-\min \left(X_{1 j}, X_{2 j}, L, X_{n j}\right)}{\max \left(X_{1 j}, X_{2 j}, L, X_{n j}\right)-\min \left(X_{1 j}, X_{2 j}, L, X_{n j}\right)}, \\
i=1,2, L, n ; j=1,2, L, p .
\end{array}
$$

When $\mathrm{X} i \mathrm{i}$ is a negative indicator [31],

$$
\begin{array}{r}
X_{i j}^{*}=\frac{\max \left(X_{1 j}, X_{2 j}, L, X_{n j}\right)-X_{i j}}{\max \left(X_{1 j}, X_{2 j}, L, X_{n j}\right)-\min \left(X_{1 j}, X_{2 j}, L, X_{n j}\right)}, \\
i=1,2, n ; L j=1,2, L p,
\end{array}
$$

where $i$ represents the sample; $j$ represents the indicator, $X_{i j}^{\prime}$ represents the standardized value of the $i$ th sample under the $j$ indicator, $\min \left(X_{1 j}, X_{2 j}, L, X_{n j}\right)$ represents the minimum value in the $j$ indicator value, and $\max \left(X_{1 j}, X_{2 j}, L, X_{n j}\right)$ represents the maximum value in the $j$ indicator value.

Normalized data after calculation:

$$
X^{*}=\left[\begin{array}{cccc}
x_{11}^{*} & x_{12}^{*} & \cdots & x_{1 p}^{*} \\
x_{21}^{*} & x_{22}^{*} & \cdots & x_{2 p}^{*} \\
\vdots & \vdots & & \vdots \\
x_{n 1}^{*} & x_{n 2}^{*} & \cdots & x_{n p}^{*}
\end{array}\right]=\left(X_{1}^{*}, X_{2}^{*}, \cdots, X_{p}^{*}\right)
$$

(b) Computation of entropy value [31]:

$$
e_{j}=-k \sum_{i=1}^{n} p_{i j} \ln p_{i j}
$$

where $e_{j}$ represents the entropy value of index $j ; k>0$; $p_{i j}=x_{i j}^{*} / \sum_{n}^{i=1} x_{i j}^{*}$ represents the weight of scheme $i$ under index $j ; n$ represents the number of samples.

(c) Computation of the different coefficient [31]:

$$
g_{j}=1-e_{j}
$$

where $g_{j}$ represents the different coefficient of index $j$ and $e_{j}$ is the same as in (5) above.

(d) Computation of the index weight [31]:

$$
a_{j}=\frac{g_{j}}{\sum_{j=1}^{n} g_{j}},
$$

where $a_{j}$ represents the weight of index $j$ and $g_{j}$ is the same as in (6) above.

Through ArcGIS software, establish the raster layer of each index, use the above entropy value calculation formula, and use the raster calculator to calculate the vulnerability to obtain the vulnerability value of the mountain settlement. At the same time, in order to conduct a comparative analysis of the vulnerability of mountain settlements in the upper Min River in different periods, the results of mountain settlement evaluations in 2006, 2009, and 2005 were subjected to dispersion standardization (Min-Max standardization). So that the vulnerability index of each period is changed to between $[0,1]$. The vulnerability level is divided into 5 categories using the equal division method, which are in turn, low (0.0-0.2), comparatively low (0.2-0.4), medium (0.4-0.6), comparatively high (0.6-0.8), and high (0.8-1.0) five mountain settlement vulnerable areas. The standardized transfer function for dispersion is as follows [31]:

$$
X^{\prime}=\frac{X-\min }{\max -\min }
$$

where $X^{\prime}$ is the normalized value of the sample data, $X$ is the original value of the sample data, max is the maximum value of the sample data, and min is the minimum value of the sample data. 


\section{Results and Analysis}

4.1. Computation and Analysis of Vulnerability Indicators. The weights of various indexes changed greatly in 2006, 2009, and 2015 (Table 2). In 2006, the weights of road density, economic density, building coverage, and population density were larger, and the weight of per capita GDP and general public budget expenditure was lower. In 2009, the weights of building coverage and road density were higher, and the weight of per capita GDP, general public budget expenditure, and number of medical technicians per 10,000 persons were lower. In 2015, the weights of building coverage and highway density were higher, while the weights of general public budget expenditure, per capita mobile phones, and the number of medical technicians per 10,000 persons were lower.

In the evaluation process using the entropy method, the indicators with too small weights can be excluded according to the size of the entropy weight, in order to facilitate a more accurate and reliable evaluation [27]. According to Table 2, teacher-student ratio, number of village committees per 10,000 persons general public budget expenditure, number of medical technicians per 10,000 persons, and engineering protection with $a_{j}<0.03$ should be excluded. The 11 indicators finally selected property threatened by geological disasters (N1), the number of people threatened by geological disasters (N2), population density (N3), hazard density (N4), economic density (N5), GDP ratio of primary industry (N6), building coverage (N7), road density (N8), per capita GDP (N9), per capital investment in fixed assets (N10), and per capita mobile phones (N11). Finally, calculate the weight of each indicator in 2006, 2009, and 2015 again (Table 3).

4.2. Results of Vulnerability Assessment and Its Dynamic Changes. As shown in Table 4, the medium and low vulnerability areas accounted for the highest proportion between 2006 and 2015. The vulnerable areas changed from medium to low vulnerable areas, which indicates that the implementation of risk-aversion relocation and land-change relocation policies changed the vulnerability of the upper Min River. According to the percentage of each vulnerable area, in the highly vulnerable area, the percentage trend of vulnerable areas was $2006>2015>2009$; in the middle and comparatively high vulnerable areas, the trend was $2006>2009>2015$; and in the comparatively low and low vulnerable areas, the trend of the proportion of lower vulnerability was $2015>2009>2006$. The main reason for this variation was the evacuation and relocation projects of the Wenchuan disaster reconstruction plan that made many people move to dangerous areas.

In 2006, most settlements in Wenchuan, Mao, and Heishui counties had a high vulnerability to geological hazards-mainly due to their dense population, frequent economic activities, and more potential hazards. Comparatively, higher and middle vulnerable areas were distributed mainly on both sides of the Min River in Wenchuan, Mao, and Songpan counties. The vulnerability of geological hazards in most areas of Li County was relatively low, and the relatively high vulnerability areas were mainly near Zagunao Town. Wolong Town, and Gengda Town in Wenchuan County which are the natural reserves of the giant panda. Because of better vegetation, less human engineering activities, and sparse distribution of geological hazards and settlements, the vulnerability of geological hazards was relatively low (Figure 3(a)).

In 2009, the areas with the high and comparatively high vulnerability of geological hazards in the upper Min River were concentrated mainly in Songpan County, Heishui County, and townships on both sides of the mainstream of Min River in Mao County. Except for the high and comparatively high vulnerability in Songpan County, the upper reaches of the Heishui River Basin and the villages and towns on the right side of the river course were moderately vulnerable. The vulnerability values of the Zagunao River Basin in Li County and the Yuzixi River Basin in Wenchuan County were low as a whole, while those of the Shouxi River Basin were lower (Figure 3(b)).

In 2015, the settlement vulnerability of geological hazards in the upper Min River was relatively low (Figure 3(c)). Nevertheless, the highly vulnerable areas were mainly Fengyi Town in Mao County, Weizhou Town in Wenchuan County, and Jinan Town and Jinan Hui Township in Songpan County. These areas are mainly the government locations of the districts and counties, which are relatively low on both sides of the river and are conducive to settlement production. However, the upper Min River has deep mountains and valleys, which are prone to geological disasters and have a greater potential for vulnerability. Hongkou Township of Dujiangyan has a comparatively high vulnerability. Due to its large population and dense economy, the potential loss is enormous. Except for the high and comparatively high vulnerability in Songpan County, the upper reaches of the Heishui River Basin and the villages and towns on the right side of the river course were moderately vulnerable. The vulnerability values of the Zagunao River Basin and the Yuzixi River Basin were low as a whole.

\section{Discussion}

The main indicators of the vulnerability of geological hazards in the upper Min River were population density, building coverage, economic density, and road density. They indicate that population, buildings, and highways are the main disaster-bearing bodies of geological hazards in the upper Min River and that regional economic value is the most direct loss of disaster threat objects. Among the factors contributing less, GDP per capita and general public budget expenditure were relatively low in 2006. The reasons that accounted for this were the mountain areas in the upper Min River, poor economic foundation, lack of revenue sources for the government, low corresponding public budget expenditure, and internal differences. In 2009, per capita GDP, general public budget expenditure, and the number of medical technicians per 10,000 persons accounted for the lowest share. Compared with 2006, after the Wenchuan earthquake, there was an increase in the number of medical 
TABLE 2: Entropy value, difference coefficient, and weight of vulnerability indicators of geological hazards in the upper Min River.

\begin{tabular}{|c|c|c|c|c|c|c|c|c|c|c|}
\hline \multirow{2}{*}{ No. } & \multirow{2}{*}{ Parameter } & \multicolumn{3}{|c|}{2006} & \multicolumn{3}{|c|}{2009} & \multicolumn{3}{|c|}{2015} \\
\hline & & $e_{j}$ & $g_{j}$ & $a_{j}$ & $e_{j}$ & $g_{j}$ & $a_{j}$ & $e_{j}$ & $g_{j}$ & $a_{j}$ \\
\hline D1 & Population density & 0.7702 & 0.2298 & 0.1322 & 0.8424 & 0.1576 & 0.1161 & 0.8422 & 0.1578 & 0.1028 \\
\hline D2 & Building coverage & 0.7645 & 0.2355 & 0.1354 & 0.7287 & 0.2713 & 0.1999 & 0.6045 & 0.3955 & 0.2577 \\
\hline D3 & Economic density & 0.6607 & 0.3393 & 0.1951 & 0.8590 & 0.1410 & 0.1039 & 0.7773 & 0.2227 & 0.1451 \\
\hline D4 & Per capita GDP & 0.9850 & 0.0150 & 0.0086 & 0.9950 & 0.0050 & 0.0037 & 0.9843 & 0.0157 & 0.0102 \\
\hline D5 & GDP ratio of primary ind & 0.9323 & 0.0677 & 0.0389 & 0.9406 & 0.0594 & 0.0438 & 0.8754 & 0.1246 & 0.0812 \\
\hline D6 & Per capita investment in fixed assets & 0.9513 & 0.0487 & 0.0280 & 0.9189 & 0.0811 & 0.0598 & 0.9818 & 0.0182 & 0.0119 \\
\hline D7 & General public budget expenditure & & 0.0036 & 0.0021 & 0.9946 & 0.0054 & 0.0040 & 0.9963 & 0.0037 & 0.0024 \\
\hline D8 & Road density & 0.6671 & 0.3329 & 0.1914 & 0.7637 & 0.2363 & 0.1741 & 0.7896 & 0.2104 & 0.1371 \\
\hline D9 & Number of village committees per 10,000 pe & 0.9764 & 0.0236 & 0.0135 & 0.9828 & 0.0172 & 0.0127 & 0.9801 & 0.0199 & 0.0130 \\
\hline D10 & Number of medical technicians per 10,000 persons & 0.9680 & 0.0320 & 0.0184 & 0.9901 & 0.0099 & 0.0073 & 0.9935 & 0.0065 & 0.0042 \\
\hline D11 & Teacher-student ratio & 0.9603 & & 0.0228 & 0.9744 & 0.0256 & 0.0189 & 0.9510 & 0.0490 & 0.0319 \\
\hline D12 & Per capita mobile phone & 0.9439 & 0.0561 & 0.0323 & 0.9551 & 0.0449 & 0.0331 & 0.9919 & 0.0081 & 0.0053 \\
\hline D13 & Hazard density & 0.8955 & 0.1045 & 0.0601 & 0.8932 & 0.1068 & 0.0787 & 0.8932 & 0.1068 & 0.0696 \\
\hline D14 & e threatened by ge & 0.8904 & 0.1096 & 0.0630 & 0.9053 & 0.0947 & 0.0698 & 0.9053 & 0.0947 & 0.0617 \\
\hline D15 & Property threatened by geological disasters & 0.9036 & 0.0964 & 0.0554 & 0.9039 & 0.0961 & 0.0708 & 0.9039 & 0.0961 & 0.0626 \\
\hline D16 & Engineering protection & 0.9952 & 0.0048 & 0.0028 & 0.9952 & 0.0048 & 0.0036 & 0.9952 & 0.0048 & 0.0031 \\
\hline
\end{tabular}

TABLE 3: Final weight of vulnerability indicators of geological hazards in the upper Min River.

\begin{tabular}{ccccccccccccc}
\hline & & N1 & N2 & N3 & N4 & N5 & N6 & N7 & N8 & N9 & N10 & N11 \\
\hline \multirow{4}{*}{$a_{j}$} & 2006 & 0.0590 & 0.0670 & 0.1405 & 0.0639 & 0.2074 & 0.0414 & 0.1440 & 0.2035 & 0.0092 & 0.0298 & 0.0343 \\
& 2009 & 0.0743 & 0.0732 & 0.1218 & 0.0825 & 0.1090 & 0.0459 & 0.2096 & 0.1826 & 0.0038 & 0.0627 & 0.0347 \\
& 2015 & 0.0663 & 0.0653 & 0.1088 & 0.0736 & 0.1535 & 0.0859 & 0.2726 & 0.1450 & 0.0108 & 0.0126 & 0.0056 \\
\hline
\end{tabular}

TABLE 4: Vulnerability zoning results of geological hazards in the upper Min River for 2006, 2009, and 2015.

\begin{tabular}{|c|c|c|c|c|c|c|}
\hline \multirow{2}{*}{ Vulnerability zoning } & \multicolumn{2}{|c|}{2006} & \multicolumn{2}{|c|}{2009} & \multicolumn{2}{|c|}{2015} \\
\hline & Area $\left(\mathrm{km}^{2}\right)$ & Area (\%) & Area $\left(\mathrm{km}^{2}\right)$ & Area (\%) & Area $\left(\mathrm{km}^{2}\right)$ & Area (\%) \\
\hline High & 142.1663 & 14.88 & 56.8283 & 6.98 & 61.7794 & 13.35 \\
\hline Comparatively high & 101.2278 & 10.59 & 169.2009 & 20.78 & 31.2595 & 6.75 \\
\hline Middle & 440.9356 & 46.14 & 325.557 & 39.99 & 72.7635 & 15.72 \\
\hline Comparatively low & 177.2316 & 18.54 & 139.7377 & 17.16 & 234.8497 & 50.73 \\
\hline Low & 94.1431 & 9.85 & 122.7882 & 15.08 & 62.2737 & 13.45 \\
\hline
\end{tabular}

technicians per 10,000 persons, showing higher medical and health investment by the government in remote areas. As a result, the regional differences in the distribution of doctors and technicians narrowed. More importantly, although the share of general public budget expenditure was still small, it was quite different from the situation in 2006. The government still invested less in the area in 2006, but the state invested a lot of money after the Wenchuan earthquake, and with support from all parts of the country, the general public budget input in 2009 was very high compared with that in 2006.

Compared with 2006 and 2009, in 2015, the weight of per capita mobile phones was relatively lower. The study area still had mainly fixed phones and functional computers in 2006 and 2009, and the mobile infrastructure was not perfect. With imperfection in mobile infrastructure, there was a big difference in the per capita telephones in each town. In 2015, due to the government's construction of basic communication facilities in the region, coupled with the popularization of $3 \mathrm{G}$ and $4 \mathrm{G}$ signals, smartphones entered thousands of mountain families, and now almost every household has a mobile phone.

The average vulnerability of villages and towns in the upper Min River was 0.2213 in 2006, 0.2290 in 2009, and 0.2251 in 2015 . The order of the vulnerability value was $2009>2015>2006$, and the overall vulnerability of the upper Min River was low. The main reason for the highest 

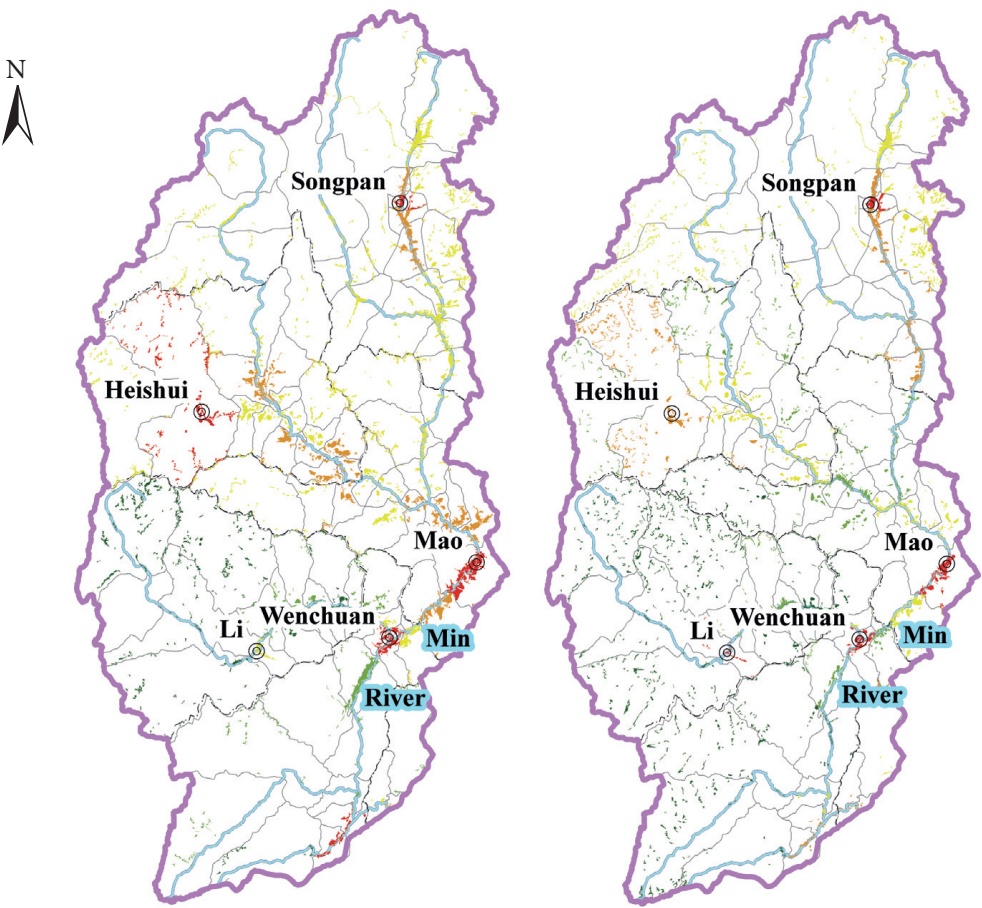

$\begin{array}{llll}0 & 15 & 30 & 60 \mathrm{~km}\end{array}$

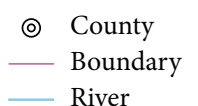

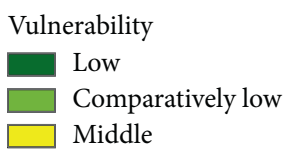

(b)

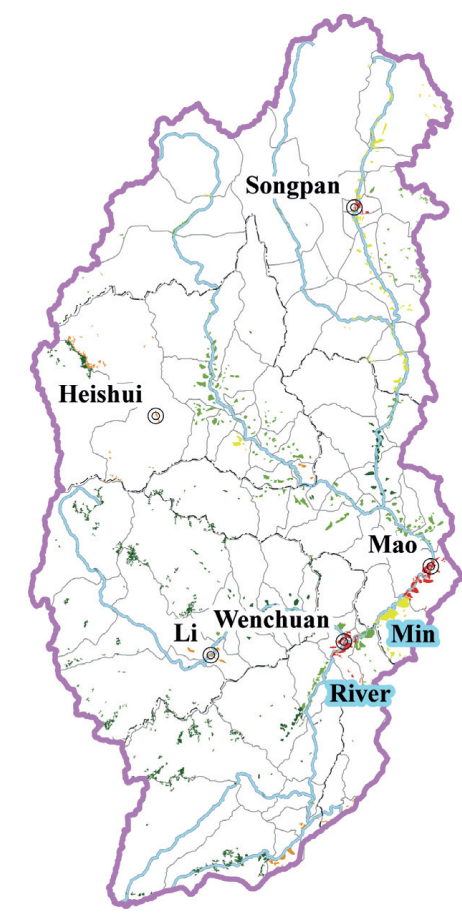

Comparatively high

High

(c)

FIGURE 3: Vulnerability zoning of geological hazards in the upper Min River for several years. (a) 2006. (b) 2009. (c) 2015.

vulnerability value in the area in 2009 was earthquakestricken areas of Wenchuan, where production and life were seriously affected by earthquakes and the secondary geological hazards caused by the earthquake.

The vulnerability value in 2015 was much lower than that in 2009, but it was still higher than that in 2006, which indicates that the reconstruction effect of the Wenchuan earthquake was remarkable, but there was a need for strengthening disaster prevention and mitigation mechanism. Before 2015, five counties in the upper Min River were deeply poverty-stricken (among them Heishui County was a state-level poverty-stricken county). With the deepening implementation of the precise poverty alleviation policy, the economic development of the region has been rapid in the past 10 years. Especially after 2008, the national postdisaster reconstruction has invested a lot of manpower and material resources in the region, bringing Mao, Wenchuan, and Li counties out of poverty successively in 2017, which is consistent with the results of vulnerability study.
According to the ascending order of vulnerability values of 84 townships in the upper Min River in 2006, 2009, and 2015 (Table 5), the time-varying curve of settlement vulnerability of geological hazards can be obtained (Figure 4). From the trend of change, the vulnerability curve is 76-78 in villages and towns, which divides the whole area into two parts: high value and low-value areas. The vulnerability trend of high value area is $2009>2015>2006$, and that of low value area is $2009>2006>2015$. In each year, the proportion of towns in the low-value areas is higher, while that of the highvalue area is lower. Vulnerability rises faster in the high-value area and slower in the low-value area, which is like the "Matthew effect" in economics. This special "Matthew effect" can better reflect the changing trend of vulnerability on a time scale. That is, the settlement vulnerability of geological hazards has polarized-the areas with high vulnerability value continue to rise and the areas with low vulnerability value continue to decrease. This also indirectly indicates that we should focus on building a disaster prevention and emergency response capacity in highly vulnerable areas. 
TABLE 5: Vulnerability of townships to geological hazards in the upper Min River for 2006, 2009, and 2015.

\begin{tabular}{|c|c|c|c|c|c|c|}
\hline No. & Vulnerability in 2006 & Township & Vulnerability in 2009 & Township & Vulnerability in 2015 & Township \\
\hline 1 & 0.0000 & Jiabi & 0.0000 & Jiabi & 0.0000 & Jiabi \\
\hline 2 & 0.0268 & Miyaluo & 0.0390 & Sanjiang & 0.0211 & Miyaluo \\
\hline 3 & 0.0362 & Guergou & 0.0459 & Miyaluo & 0.0398 & Guergou \\
\hline 4 & 0.0393 & Shangmeng & 0.0526 & Shangmeng & 0.0419 & Shangmeng \\
\hline 5 & 0.0545 & Puxi & 0.0572 & Guergou & 0.0488 & Puxi \\
\hline 6 & 0.0623 & Putou & 0.0597 & Gengda & 0.0570 & Weicheng \\
\hline 7 & 0.0634 & Tonghua & 0.0649 & Yinxing & 0.0610 & Songpinggou \\
\hline 8 & 0.0638 & Sanjiang & 0.0821 & Putou & 0.0664 & Putou \\
\hline 9 & 0.0924 & Yinxing & 0.0829 & Weicheng & 0.0672 & Sanjiang \\
\hline 10 & 0.0960 & Gengda & 0.0860 & Puxi & 0.0721 & Tonghua \\
\hline 11 & 0.0971 & Taoping & 0.0955 & Tonghua & 0.0755 & Diexi \\
\hline 12 & 0.1022 & Xuecheng & 0.1019 & Wolong & 0.0766 & Yinxing \\
\hline 13 & 0.1042 & Xiameng & 0.1062 & Songpinggou & 0.0813 & Wolong \\
\hline 14 & 0.1073 & Wolong & 0.1102 & Diexi & 0.0819 & Gengda \\
\hline 15 & 0.1129 & Ganbao & 0.1163 & Taoping & 0.0850 & Wadi \\
\hline 16 & 0.1172 & Muka & 0.1197 & Longxi & 0.0851 & Kalong \\
\hline 17 & 0.1223 & Caopo & 0.1218 & Xiameng & 0.0879 & Taoping \\
\hline 18 & 0.1319 & Xiabazhai & 0.1305 & Ganbao & 0.0951 & Taiping \\
\hline 19 & 0.1350 & Yanyun & 0.1327 & Shidaguan & 0.0984 & Sanlong \\
\hline 20 & 0.1368 & Weicheng & 0.1353 & Xuecheng & 0.0997 & Caopo \\
\hline 21 & 0.1404 & Shangbazhai & 0.1377 & Heihu & 0.1007 & Muka \\
\hline 22 & 0.1408 & Longxi & 0.1396 & Wadi & 0.1017 & Shidaguan \\
\hline 23 & 0.1439 & Hongzha & 0.1412 & Kalong & 0.1017 & Xiameng \\
\hline 24 & 0.1442 & Songpinggou & 0.1424 & Caopo & 0.1026 & Xuecheng \\
\hline 25 & 0.1446 & Yanmen & 0.1424 & Sanlong & 0.1035 & Heihu \\
\hline 26 & 0.1493 & Caoyuan & 0.1624 & Qugu & 0.1040 & Shashiduo \\
\hline 27 & 0.1497 & Yingxiu & 0.1626 & Yadu & 0.1075 & Ganbao \\
\hline 28 & 0.1500 & Dazhai & 0.1626 & Shashiduo & 0.1171 & Longxi \\
\hline 29 & 0.1502 & Shuijing & 0.1628 & Taiping & 0.1185 & Yanmen \\
\hline 30 & 0.1504 & Mouni & 0.1653 & Baixi & 0.1198 & Luoduo \\
\hline 31 & 0.1609 & Shanba & 0.1737 & Yanmen & 0.1249 & Qugu \\
\hline 32 & 0.1656 & Diexi & 0.1739 & Muka & 0.1265 & Yadu \\
\hline 33 & 0.1685 & Taiping & 0.1764 & Luoduo & 0.1291 & Baixi \\
\hline 34 & 0.1698 & Miansi & 0.1800 & Ciba & 0.1291 & Ciba \\
\hline 35 & 0.1705 & Shashiduo & 0.1806 & Miansi & 0.1343 & Longba \\
\hline 36 & 0.1709 & Hongkou & 0.1861 & Longba & 0.1407 & Huilong \\
\hline 37 & 0.1712 & Daxing & 0.1905 & Yanyun & 0.1412 & Yingxiu \\
\hline 38 & 0.1754 & Heihu & 0.1962 & Shangbazhai & 0.1421 & Zhimulin \\
\hline 39 & 0.1783 & Hongtu & 0.1990 & Goukou & 0.1423 & Zhawo \\
\hline 40 & 0.1790 & Wadi & 0.1994 & Hongzha & 0.1461 & Waboliangzi \\
\hline 41 & 0.1819 & Shidaguan & 0.1994 & Xiabazhai & 0.1467 & Qinglang \\
\hline 42 & 0.1823 & Sanlong & 0.2009 & Zhimulin & 0.1530 & Mawo \\
\hline 43 & 0.1834 & Xiaoxing & 0.2023 & Waboliangzi & 0.1552 & Keku \\
\hline 44 & 0.1888 & Kalong & 0.2033 & Shuijing & 0.1576 & Goukou \\
\hline 45 & 0.1890 & Mawo & 0.2037 & Mouni & 0.1632 & Shidiaolou \\
\hline 46 & 0.1915 & Luoduo & 0.2039 & Qinglang & 0.1764 & Shuangliusuo \\
\hline 47 & 0.1953 & Ciba & 0.2063 & Shidiaolou & 0.1828 & Weigu \\
\hline 48 & 0.1964 & Zhenping & 0.2112 & Nanxin & 0.1852 & Hongyan \\
\hline 49 & 0.1968 & Baixi & 0.2114 & Zhawo & 0.1853 & Feihong \\
\hline 50 & 0.1968 & Longba & 0.2116 & Caoyuan & 0.2044 & Weimen \\
\hline 51 & 0.2042 & Keku & 0.2128 & Shanba & 0.2193 & Musu \\
\hline 52 & 0.2064 & Yadu & 0.2148 & Dazhai & 0.2538 & Miansi \\
\hline 53 & 0.2064 & Longchi & 0.2267 & Huilong & 0.2578 & Hongkou \\
\hline 54 & 0.2084 & Qinglang & 0.2278 & Hongtu & 0.2672 & Xiabazhai \\
\hline 55 & 0.2131 & Chuanzhusi & 0.2298 & Mawo & 0.2710 & Longchi \\
\hline 56 & 0.2149 & Shuangliusuo & 0.2306 & Yingxiu & 0.2721 & Hongzha \\
\hline 57 & 0.2162 & Zhawo & 0.2306 & Daxing & 0.2721 & Yanyun \\
\hline 58 & 0.2162 & Zhimulin & 0.2322 & Feihong & 0.2750 & Dazhai \\
\hline 59 & 0.2180 & Minjiang & 0.2354 & Keku & 0.2752 & Shangbazhai \\
\hline 60 & 0.2241 & Qugu & 0.2401 & Xiaoxing & 0.2794 & Shuijing \\
\hline
\end{tabular}


TABLE 5: Continued.

\begin{tabular}{|c|c|c|c|c|c|c|}
\hline No. & Vulnerability in 2006 & Township & Vulnerability in 2009 & Township & Vulnerability in 2015 & Township \\
\hline 61 & 0.2245 & Hongyan & 0.2437 & Hongyan & 0.2817 & Mouni \\
\hline 62 & 0.2299 & Shidiaolou & 0.2510 & Weigu & 0.2835 & Daxing \\
\hline 63 & 0.2323 & Shili Hui & 0.2545 & Shuangliusuo & 0.2886 & Shanba \\
\hline 64 & 0.2325 & Zhenjiangguan & 0.2547 & Seergu & 0.2897 & Caoyuan \\
\hline 65 & 0.2328 & Goukou & 0.2597 & Weimen & 0.3040 & Hongtu \\
\hline 66 & 0.2354 & Waboliangzi & 0.2636 & Minjiang & 0.3062 & Xiaoxing \\
\hline 67 & 0.2368 & Anhong & 0.2660 & Zhenping & 0.3177 & Zhenping \\
\hline 68 & 0.2439 & Huilong & 0.2769 & Chuanzhusi & 0.3200 & Minjiang \\
\hline 69 & 0.2546 & Qingyun & 0.2797 & Anhong & 0.3293 & Nanxin \\
\hline 70 & 0.2571 & Musu & 0.2807 & Musu & 0.3411 & Zhenjiangguan \\
\hline 71 & 0.2716 & Weigu & 0.2809 & Zhenjiangguan & 0.3458 & Anhong \\
\hline 72 & 0.2745 & Zagunao & 0.3028 & Shili Hui & 0.3467 & Chuanzhusi \\
\hline 73 & 0.2825 & Feihong & 0.3087 & Hongkou & 0.3542 & Zipingpu \\
\hline 74 & 0.2825 & Seergu & 0.3204 & Shuimo & 0.3609 & Qingyun \\
\hline 75 & 0.3040 & Weimen & 0.3289 & Qingyun & 0.3616 & Xuankou \\
\hline 76 & 0.3129 & Nanxin & 0.3461 & Luhua & 0.3699 & Shili Hui \\
\hline 77 & 0.3930 & Shuimo & 0.3475 & Longchi & 0.3870 & Seergu \\
\hline 78 & 0.5282 & Jinan Hui & 0.4193 & Xuankou & 0.3874 & Shuimo \\
\hline 79 & 0.6186 & Luhua & 0.4935 & Zipingpu & 0.5389 & Luhua \\
\hline 80 & 0.6978 & Jinan & 0.5829 & Jinan Hui & 0.6135 & Zagunao \\
\hline 81 & 0.7608 & Fengyi & 0.6386 & Zagunao & 0.8388 & Fengyi \\
\hline 82 & 0.7730 & Weizhou & 0.8669 & Jinan & 0.8412 & Jinan Hui \\
\hline 83 & 0.8081 & Zipingpu & 0.9191 & Weizhou & 0.9766 & Jinan \\
\hline 84 & 1.0000 & Xuankou & 1.0000 & Fengyi & 1.0000 & Weizhou \\
\hline
\end{tabular}

The bold text indicates the transition zone from low-value vulnerability area to high-value area.

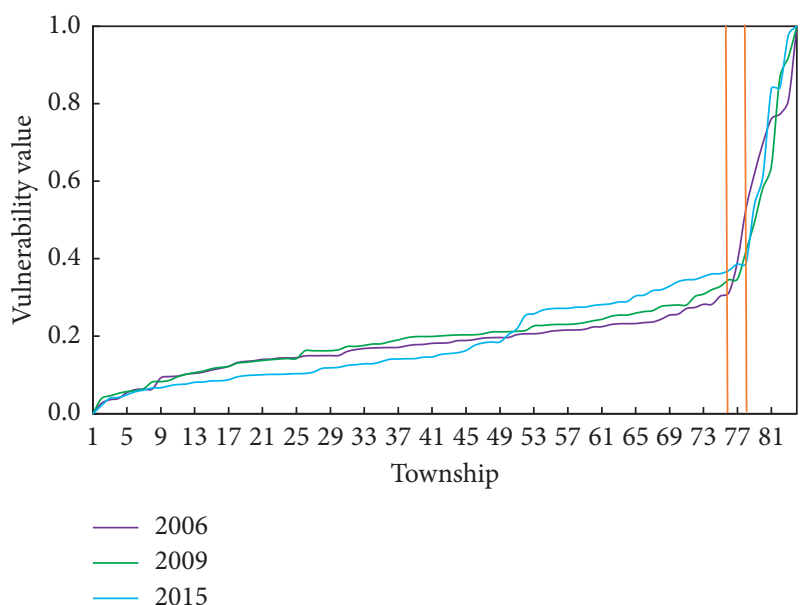

FIGURE 4: Vulnerability change of geological hazards in the upper Min River.

\section{Conclusions}

Vulnerability, as a part of risk assessment, is an indispensable part of regional risk control. Wenchuan earthquake, geological hazards, settlement migration, socioeconomic conditions, and ethnic cultural changes have a profound impact on the mountain settlement vulnerability of geological hazards in the upper Min River. The migration of mountain settlements in this area varies greatly. Due to national policy, the area of settlements has been drastically reduced, and the population living in traditional farming and animal husbandry has gradually moved to cities. As main destinations of settlement migration, the county towns of each district and county should strengthen the capacity building of disaster prevention and emergency response.

The contribution share of each index is different in different years. Population density, building coverage, economic density, and road density are the main factors affecting the settlement vulnerability of geological hazards in the upper Min River. Therefore, strengthening the construction of the regional population, economy, and infrastructure is conducive to reducing the vulnerability of the area.

The settlement vulnerability of geological hazards in the upper Min River is witnessing a dynamic change, with the highest vulnerability in 2009, the second in 2015, and the lowest in 2006. Although the Wenchuan earthquake caused considerable damage in the area, significant achievements have also been made in the postearthquake reconstruction. The time fluctuation of vulnerability distribution in villages and towns is consistent. The trend of vulnerability distribution is basically in line with the "Matthew effect." The areas with high vulnerability to settlement geological hazards will continue to increase. Therefore, we should focus on the prevention of geological hazards, the creation of an investment in reconstruction, and building emergency capacity in highly vulnerable areas.

\section{Data Availability}

The vulnerability evaluation data and relative weights used to support the findings of this study are available from the corresponding author upon request. 


\section{Conflicts of Interest}

The authors declare that there are no conflicts of interest regarding the publication of this paper.

\section{Acknowledgments}

This research was financially supported by the National Key Research and Development Program (Grant no. 2018YFC1505402), National Natural Science Foundation of China (Grant no. 41871174), the project of Science and Technology Department of Sichuan Province (Grant no. 2020YFSY0013), Opening Fund of State Key Laboratory of Geohazard Prevention and Geoenvironment Protection (Chengdu University of Technology) (Grant no. SKLGP2019K018), and the Fundamental Research Funds for the Central Universities (Grant no. 2682019CX19).

\section{References}

[1] G. J. Chen, Y. P. Fang, Y. Chen et al., A Report on the Development of Mountain Areas in China: A Study of Mountain Settlements, The Commercial Press, Beijing, China, 2007.

[2] K. N. Westgate and P. O'Keefe, “The human and social implications of earthquake risk for developing countries: towards an integrated mitigation strategy," Intergovernmental Conference on the Assessment and Mitigation of Earthquake Risk, UNESCO, Paris, France, 1976.

[3] S. L. Cutter, "Vulnerability to environmental hazards," Progress in Human Geography, vol. 20, no. 4, pp. 529-539, 1996.

[4] G. White, "Knowing better and losing ever more: the use of knowledge in hazards management," Environmental Hazards, vol. 3, no. 3-4, pp. 81-92, 2001.

[5] M. Panizza, Environmental Geomorphology, Elsevier, Amsterdam, Netherlands, 1996.

[6] R. E. Deyle, S. P. French, R. B. Olshansky et al., "Hazard assessment: the factual basis for planning and mitigation," Cooperating with Nature: Confronting Natural Hazards with Land Use Planning for Sustainable Communities, vol. 119-166, 1998.

[7] Q. Guo, "Analysis on the relations between social vulnerability and natural disaster," Exploration of Nature, vol. 16, no. 2, pp. 86-90, 1997.

[8] Z. Hu and Z. M. Sun, "Influence of social factors on disasters," Journal of Catastrophology, vol. 13, no. 1, pp. 88-91, 1998.

[9] X. L. Liu and D. W. Mo, "Site-specific debris flow vulnerability assessment," Geographical Research, vol. 21, no. 5, pp. 569$577,2002$.

[10] X. L. Liu, D. W. Mo, and X. D. Wang, "Regional vulnerability assessment of debris flows," The Chinese Journal of Geological Hazard and Control, vol. 12, no. 2, pp. 7-12, 2001.

[11] X. L. Pang, C. F. Huang, and Y. J. Zhang, "A basic mode for dynamic risk assessment of natural disaster," Journal of Catastrophology, vol. 31, no. 1, pp. 1-6, 2016.

[12] Y. L. Wang, J. Ding, D. W. Wang et al., "Assessment of the vulnerability of social economy by geological hazards in Kangding county town, western Sichuan," Sedimentary Geology and Tethyan Geology, vol. 26, no. 2, pp. 89-91, 2006.

[13] C. Tang, "A study on compilation of landslide risk map," Journal of Natural Disasters, vol. 13, no. 3, pp. 8-12, 2004.

[14] Y. P. Wu, H. M. Tang, and W. Jiang, "Landslide hazard risk prediction system for the new Badong county seat based on GIS," Hydrogeology \& Engineering Geology, no. S1, pp. 117121, 2003.
[15] C. Miao and M. Ding, "Social vulnerability assessment of geological hazards based on entropy method in Lushan earthquake-stricken area," Arabian Journal of Geosciences, vol. 8, no. 12, pp. 10241-10253, 2015.

[16] W. Q. Zhao, "The evaluation systematic index of social vulnerability to natural hazards: a case study of Chongqing city," Master's Degree thesis, Chongqing Normal University, Chongqing, China, 2008.

[17] Y. Yan, Y. Cui, J. Guo, S. Hu, Z. Wang, and S. Yin, "Landslide reconstruction using seismic signal characteristics and numerical simulations: case study of the 2017 " 6.24 " Xinmo landslide," Engineering Geology, vol. 270, no. 5, p. 105582, 2020.

[18] M. T. Ding, T. Huang, H. Zheng, and G. H Yang, "Respective influence of vertical mountain differentiation on debris flow occurrence in the Upper Min River, China," Scientific Reports, vol. 10, p. 11689, 2020.

[19] Geo-Environmental Information System of Sichuan Province, http://202.61.89.33:16003.

[20] O. Hungr, S. Leroueil, and L. Picarelli, "The Varnes classification of landslide types, an update," Landslides, vol. 11, no. 2, pp. 167-194, 2014.

[21] M. Ding, M. Heiser, J. Hübl, and S. Fuchs, "Regional vulnerability assessment for debris flows in China-a CWS approach," Landslides, vol. 13, no. 3, pp. 537-550, 2016.

[22] J. Fan, Assessment Guidelines for Resources and Environmental Carrying Capacity and Territorial Development Suitability, Science Press, Beijing, China, 2019.

[23] F. Guzzetti, A. Carrara, M. Cardinali, and P. Reichenbach, "Landslide hazard evaluation: a review of current techniques and their application in a multi-scale study, Central Italy," Geomorphology, vol. 31, no. 1-4, pp. 181-216, 1999.

[24] S. L. Cutter, B. J. Boruff, and W. L. Shirley, "Social vulnerability to environmental hazards*," Social Science Quarterly, vol. 84, no. 2, pp. 242-261, 2003.

[25] S. L. Cutter and C. Finch, "Temporal and spatial changes in social vulnerability to natural hazards," Proceedings of the National Academy of Sciences, vol. 105, no. 7, pp. 2301-2306, 2008.

[26] M. Ding and T. Huang, "Vulnerability assessment of population in mountain settlements exposed to debris flow: a case study on Qipan gully, Wenchuan County, China," Natural Hazards, vol. 99, no. 1, pp. 553-569, 2019.

[27] National Earth System, Science Data Sharing Infrastructure, http://www.geodata.cn.

[28] K. H. Qiu, Management Decision and Applied Entropy, China Machine Press, Beijing, China, 2002.

[29] A. G. Zhou, J. W. Zhou, H. C. Liang et al., Geological Environment Evaluation, China University of Geosciences Press, Wuhan, China, 2008.

[30] C. Miao and M. T. Ding, "Comparative analysis of social vulnerability of seismic areas: a case study of Lushan earthquake and Ludian earthquake," China Safety Science Journal, vol. 27, no. 4, pp. 169-174, 2017.

[31] G. J. Chen, C. C. Ding, and Q. R. Guo, "Empirical research on the relationship between new industrialization, new urbanization and rural rejuvenation level based on entropy weighting method," Social Sciences in Hunan, vol. 6, pp. 114-124, 2018.

[32] J. X. Ding, S. H. Zhou, Z. F. Yang, Y. J Shang, and J. T. Yin, "Quantitative assessment of geological hazards in segment between Ranwu and Dongjiu in Sichuan-Tibet highway: presentation and application of concept of geological hazard entropy," Journal of Natural Disasters, vol. 14, no. 4, pp. 79-84, 2005. 